\title{
2016 update of the EULAR recommendations for the management of early arthritis
}

\author{
Bernard Combe, ${ }^{1}$ Robert Landewe ${ }_{1}{ }^{2}$ Claire I Daien, ${ }_{1}^{1}$ Charlotte Hua, ${ }_{1}^{1}$ Daniel Aletaha ${ }_{1}{ }^{3}$ \\ Jose María Álvaro-Gracia, ${ }^{4}$ Margôt Bakkers, ${ }^{5}$ Nina Brodin, ${ }^{6,7}$ Gerd R Burmester, ${ }^{8}$ \\ Catalin Codreanu ${ }_{1}^{9}$ Richard Conway, ${ }^{10}$ Maxime Dougados, ${ }_{11}$ Paul Emery, ${ }^{12}$

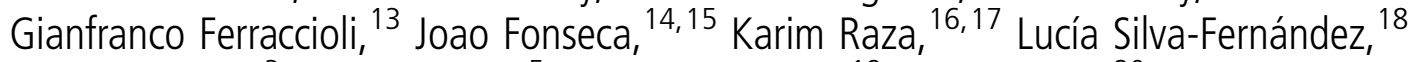 \\ Josef S Smolen, ${ }^{3}$ Diana Skingle, ${ }^{5}$ Zoltan Szekanecz, ${ }_{1}^{19}$ Tore K Kvien, ${ }^{20}$ \\ Annette van der Helm-van Mil, ${ }^{21,22}$ Ronald van Vollenhoven ${ }^{23}$
}

\section{Handling editor Hans WJ Bijlsma \\ For numbered affiliations see end of article.}

\section{Correspondence to} Professor Bernard Combe, Rheumatology Department, Lapeyronie Hospital, Montpellier University, Montpellier cedex 5 34295, France; b-combe@ chu-montpellier.fr

$B C$ and $R L$ are joint first authors

Received 27 September 2016 Accepted 24 November 2016 Published Online First 15 December 2016

\section{SLinked}

- http://dx.doi.org/10.1136/ annrheumdis-2016-211005

\section{CrossMark}

To cite: Combe $B$ Landewe $\mathrm{R}$, Daien $\mathrm{Cl}$, et al. Ann Rheum Dis 2017;76:948-959.

\section{ABSTRACT}

Objectives Since the 2007 recommendations for the management of early arthritis have been presented, considerable research has been published in the field of early arthritis, mandating an update of the 2007

European League Against Rheumatism (EULAR) recommendations for management of early arthritis.

Methods In accordance with the 2014 EULAR Standardised Operating Procedures, the expert committee pursued an approach that was based on evidence in the literature and on expert opinion. The committee involved 20 rheumatologists, 2 patients and 1 healthcare professional representing 12 European countries. The group defined the focus of the expert committee and target population, formulated a definition of 'management' and selected the research questions. A systematic literature research (SLR) was performed by two fellows with the help of a skilled librarian. A set of draft recommendations was proposed on the basis of the research questions and the results of the SLR. For each recommendation, the categories of evidence were identified, the strength of recommendations was derived and the level of agreement was determined through a voting process.

Results The updated recommendations comprise 3 overarching principles and 12 recommendations for managing early arthritis. The selected statements involve the recognition of arthritis, referral, diagnosis, prognostication, treatment (information, education, pharmacological and non-pharmacological interventions), monitoring and strategy. Eighteen items were identified as relevant for future research.

Conclusions These recommendations provide rheumatologists, general practitioners, healthcare professionals, patients and other stakeholders with an updated EULAR consensus on the entire management of early arthritis.

Peripheral inflammatory arthritis is among the most common features with which patients present in clinical rheumatology. Identifying the underlying disease can be difficult, particularly at an early stage. In clinical practice, early inflammatory arthritis is frequently undifferentiated. ${ }^{1}$ Early arthritis can develop into established rheumatoid arthritis (RA) or another definite arthropathy, can resolve spontaneously, or may remain undifferentiated for indefinite periods. To better evaluate diagnosis and outcome in arthritis, it has been proposed to first recognise inflammatory arthritis; then search for a definite diagnosis (eg, peripheral or axial spondyloarthritis; psoriatic arthritis (PsA); systemic lupus erythematosus, etc), and finally estimate the risk of developing persistent and/or erosive arthritis and propose an optimal therapeutic strategy. ${ }^{2}{ }^{3}$ Although the prognosis of early arthritis is still difficult to define, a combination of clinical, laboratory and radiographic parameters may help to predict patients' outcomes with acceptable accuracy.

The management of early arthritis has changed considerably in the past few years under the influence of new concepts for diagnosis and new effective therapies. Conventional synthetic disease-modifying antirheumatic drugs (csDMARDs) have been shown to slow disease progression in chronic inflammatory arthritides such as RA and PsA. ${ }^{4-6}$ Furthermore, biological (b) DMARDs have demonstrated rapid and sustained disease control associated with an arrest of joint destruction. ${ }^{78}$ A large body of evidence points to the usefulness of very early DMARD-start for early chronic inflammatory arthritis, preferably before the onset of erosions, in order to reduce or even prevent the risk of (further) joint damage and disability. ${ }^{5}{ }^{10}$ Also, the assessment and tight monitoring of patients with early arthritis serves to better adapt therapeutic strategies. ${ }^{911}$ Beyond doubt, the treatment goal of early arthritis should now be clinical remission and prevention of joint destruction.

Patients with early arthritis should be identified and referred to rheumatologists to confirm the presence of arthritis, the (potential) diagnosis and its prognosis and initiate appropriate treatment strategies based on these findings. Furthermore, management of early arthritis should include more than drug treatment alone, with education, shared decision making and the role of allied healthcare professionals as important themes.

A set of recommendations for the management of arthritis should address all these different aspects.

The European League Against Rheumatism (EULAR) recommendations for the management of early arthritis have been published in 2007. ${ }^{9}$ In 2010, EULAR presented recommendations for the management of RA with synthetic and biological DMARDs, which have been updated in 2013 and 
$2016 ;^{12} 13$ in addition, recommendations for the management of PsA were recently published. ${ }^{6}$ While the latter recommendations focused on the pharmacological treatments of PsA and RA, both in advanced and in early disease, the 2007 recommendations for the management of early arthritis covered the entire spectrum of management of early arthritis, including the recognition of arthritis, referral, diagnosis, prognosis, classification, information, education, non-pharmacological interventions and monitoring of the disease process as well as pharmacological treatment. The systematic literature review (SLR) that has guided the 2007 EULAR recommendations included publications up to January 2005..$^{9}$ Between 2005 and 2015, research in early arthritis has been a major focus, and many studies have appeared in the peer-reviewed literature. This literature includes - but is not limited to-topics such as diagnosis and classification criteria, window of opportunity, imaging, prognostication, treatments and therapeutic strategies.

These developments mandated an update of the existing EULAR recommendations on early arthritis, which is reported here.

\section{METHODS}

The update of the EULAR recommendations for the management of early arthritis has followed the 2014 EULAR Standardised Operating Procedures. ${ }^{14}$ The definitions (eg, management and early arthritis) of and the target populations (rheumatologists, general practitioners, medical students, healthcare professionals, patients) addressed by the 2007 expert committee $^{9}$ were considered. Briefly, the term 'management' was defined as 'all organisational, diagnostic, medical and educational procedures related to patients seeking help for arthritis of a peripheral joint' and 'early arthritis' was restricted to 'early inflammatory joint disease'.

\section{The expert committee}

The expert committee comprised 20 rheumatologists, including 2 research fellows (CID and $\mathrm{CH}$ ), 1 healthcare professional and 2 patients, from 12 European countries.

Fifteen research questions derived from the 2007 process were proposed by the convenor (BC) and the methodologist (RL), and subsequently amended and approved by the whole committee. The selected topics included recognition of arthritis, referral, diagnosis, prognostics, classification, information, education, non-pharmacological interventions, pharmacological treatments, monitoring of the disease process, strategy and prevention.

\section{Evidence-based approach}

The research questions were adjusted for further literature research if appropriate, and structured according to the Patients-Intervention-Comparator-Outcome systematic by four of the authors (CID, CH, BC, RL). Eligible study types were also defined.

A systematic search of PubMed, Medline, Embase, CINAHL and the Cochrane library was performed, with the help of a skilled librarian (Louise Falzon, Columbia University Medical Centre, USA). All articles published in English up to December 2015 were included. Abstracts from the 2014 and 2015 EULAR and American College of Rheumatology (ACR) conferences were also considered. The search was completed by a hand search and by questioning experts for additional references. The SLR process is reported in detail in two separate articles. ${ }^{15} 16$

\section{Expert opinion approach}

Each member of the expert committee obtained insight into the results of the literature search and the accompanying levels of evidence before a meeting in January 2016. During the meeting, the results of the SLR were presented to the committee in aggregated format. Three break-out groups, chaired by one expert, were formed to amend the 2007 recommendations (14; 5-8 and 9-12) and to propose new recommendations if considered appropriate. Each group then reported its proposals and wording to the entire committee for discussion and consensus, and the final formulation of the recommendations was obtained after a vote with at least $85 \%$ agreement for each item's final wording.

After the meeting the recommendations were circulated by email to all expert committee members for further minor amendments if necessary. Categories of evidence and grades of recommendations were then determined (by CID, CH, RL, BC) according to the standards of the Oxford Centre for Evidence-Based Medicine. ${ }^{17}$ To determine the level of agreement with recommendations, an anonymised email-based voting on a $0-10$ scale was performed, a vote of 0 indicating complete disagreement with a particular recommendation and 10 indicating complete agreement. The means and SDs for scores from the whole group were calculated. The recommendations are presented in box 1 and figure 1 .

\section{RESULTS}

The discussions of the expert committee resulted in 3 overarching principles and 12 recommendations (box 1) (in 2007, 12 recommendations were formulated).

\section{Overarching principles}

The expert committee considered that some of the principles on the care of patients with early arthritis are generic and should be stated first and separated from individual recommendations on diagnosis, prognosis and treatment. The committee decided unanimously on the following three overarching principles (box 1).

Principle A:

Management of early arthritis should aim at the best care and must be based on a shared decision between the patient and the rheumatologist.

The term 'best care' is obviously a major principle in medicine. The wording 'shared decision between the patient and the rheumatologist' is more than informing the patient; it rather refers to the comprehensive process of communication, knowledge exchange and achieving consensus that should lead to a treatment decision, that is, optimal from the perspectives of both patient and clinical care provider.

Principle B:

Rheumatologists are the specialists who should primarily care for patients with early arthritis.

This statement, which was part of recommendation 1 in the 2007 recommendations, was also highlighted in the EULAR recommendations for the management of $\mathrm{RA}^{14}$ and PsA. ${ }^{6}$ Its basis is evidence that patients with chronic arthritis under rheumatologists' care receive an earlier diagnosis, start treatment earlier and have better outcomes, in particular with respect to joint damage and physical function. ${ }^{18-20}$ Rheumatologists have the expertise to establish an accurate diagnosis of early arthritis, are familiar with monitoring disease activity and with the potential severity of the disease in their patients with inflammatory arthritis and are well aware of the indications, contraindications and adverse effects of specific therapies. 
Box 12016 update of the EULAR recommendations for management of early arthritis: final recommendations based on

evidence and expert opinion

Overarching principles

A. Management of early arthritis should aim at the best care and must be based on a shared decision between the patient and the rheumatologist

B. Rheumatologists are the specialists who should primarily care for patients with early arthritis

C. A definitive diagnosis in a patient with early arthritis should only be made after a careful history taking and clinical examination, which should also guide laboratory testing and additional procedures

Recommendations

1. Patients presenting arthritis (any joint swelling, associated with pain or stiffness) should be referred to, and seen by, a rheumatologist, within 6 weeks after the onset of symptoms

2. Clinical examination is the method of choice for detecting arthritis, which may be confirmed by ultrasonography

3. If a definite diagnosis cannot be reached and the patient has early undifferentiated arthritis, risk factors for persistent and/or erosive disease, including number of swollen joints, acute phase reactants, rheumatoid factor, ACPA and imaging findings, should be considered in management decisions

4. Patients at risk of persistent arthritis should be started on DMARDs as early as possible (ideally within 3 months), even if they do not fulfil classification criteria for an inflammatory rheumatologic disease

5. Among the DMARDs, methotrexate is considered to be the anchor drug and, unless contraindicated, should be part of the first treatment strategy in patients at risk of persistent disease

6. NSAIDs are effective symptomatic therapies but should be used at the minimum effective dose for the shortest time possible, after evaluation of gastrointestinal, renal and cardiovascular risks

7. Systemic glucocorticoids reduce pain, swelling and structural progression, but in view of their cumulative side effects, they should be used at the lowest dose necessary as temporary ( $<6$ months) adjunctive treatment. Intra-articular glucocorticoid injections should be considered for the relief of local symptoms of inflammation

8. The main goal of DMARD treatment is to achieve clinical remission, and regular monitoring of disease activity, adverse events and comorbidities should guide decisions on choice and changes in treatment strategies to reach this target

9. Monitoring of disease activity should include tender and swollen joint counts, patient and physician global assessments, ESR and CRP, usually by applying a composite measure. Arthritis activity should be assessed at 1-month to 3-month intervals until the treatment target has been reached. Radiographic and patient-reported outcome measures, such as functional assessments, can be used to complement disease activity monitoring

10. Non-pharmacological interventions, such as dynamic exercises and occupational therapy, should be considered as adjuncts to drug treatment in patients with early arthritis

11. In patients with early arthritis smoking cessation, dental care, weight control, assessment of vaccination status and management of comorbidities should be part of overall patient care

12. Patient information concerning the disease, its outcome (including comorbidities) and its treatment is important. Education programmes aimed at coping with pain, disability, maintenance of ability to work and social participation may be used as adjunct interventions

ACPA, anticitrullinated peptide antibodies; CRP, C reactive protein; DMARD, disease-modifying antirheumatic drug; ESR, erythrocyte sedimentation rate; EULAR, European League Against Rheumatism; NSAIDs, non-steroidal anti-inflammatory drugs.

However, the expert committee intentionally added the term 'primarily' to this statement for three reasons: (1) the management of patients with early arthritis includes the care by primary care physicians and other healthcare professionals in a multidisciplinary approach; (2) in some places care by rheumatologists is not always available and accessible. Some countries have a shortage of rheumatologists, and in such situations patients should receive treatment from other healthcare providers with experience in the care of patients with inflammatory arthritis; (3) in some countries, task shifting from rheumatologists to other healthcare professionals is actively supported in order to facilitate early access and optimal quality of care, and to make care cheaper. Such care is still primarily under the responsibility and supervision of rheumatologists, but may be provided by other care providers.

Principle C:

A definite diagnosis in a patient with early arthritis should only be made after a careful history taking and clinical examination, which should also guide laboratory testing and additional procedures.
In the 2007 recommendations, this important statement was included as bullet point 3 . It was considered that 'good clinical practice' and a 'high level of training' suffices an opinion that was entirely expert-based. The expert group was of the unanimous opinion that the statement is so generic that it represents an overarching principle rather than a recommendation. To establish a definite diagnosis in a patient with early arthritis, the group proposed that the minimum diagnostic procedures should include careful history taking and clinical examination, keeping the different possible causes of inflammatory arthritis in mind. After excluding other causes of joint swelling and pain (eg, septic arthritis, trauma, osteoarthritis, gout), particular attention should be paid to age, geographical area and travel history, number and pattern of involved joints, axial/entheseal involvement and extra-articular features (eg, eye, skin, genitourinal and gastrointestinal symptoms), including recent infections. ${ }^{1} \mathrm{~A}$ minimal laboratory testing panel was proposed in the 2007 recommendations and should include testing for $\mathrm{C}$ reactive protein (CRP)/erythrocyte sedimentation rate (ESR), full blood cell count, transaminase levels, renal function and urine analysis, 


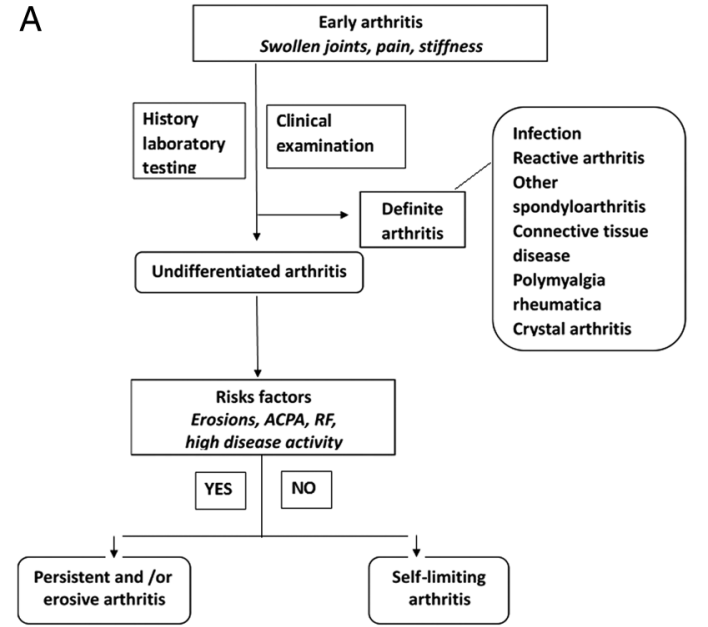

B

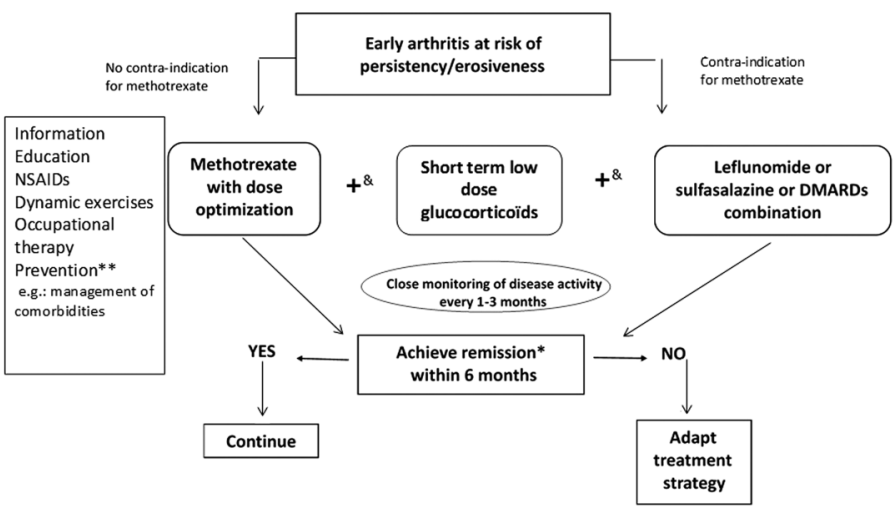

Figure 1 Algorithms based on the 2016 update of the European League Against Rheumatism recommendations for management of early arthritis. (A) Diagnosis and prognosis. (B) Treatment and strategy. \&Combination with glucocorticoids preferred. * Low disease activity could be an alternative target in rare occasions. **Should also include weight loss, smoking cessation, dental care and vaccination. ACPA, anticitrullinated peptide antibodies; DMARD, disease-modifying antirheumatic drug; NSAID, non-steroidal anti-inflammatory drug, RF, rheumatoid factor.

rheumatoid factor (RF), anticitrullinated peptide antibodies (ACPA) and antinuclear antibodies. In addition, the diagnostic procedure may be expanded with microbiology and/or serological tests (reactive arthritis, synovial fluid microbial culture, Lyme disease, parvovirus infection, hepatitis B or C), uric acid testing, synovial fluid analysis (cell count and polarised light microscopy if needed), chest and joint radiographs, but dependent on the context and the country.

\section{Recommendations}

The discussions of the expert committee culminated into 12 recommendations (box 1). In comparison with 2007, the previous recommendation 3 was transformed into overarching principle $\mathrm{C}$, while a recommendation for prevention (no. 11) was added. In addition, the order of the bullet points was slightly amended in order to better assure a logical sequence (and not for reasons of prioritisation). Table 1 displays the levels of evidence and grades for the following recommendations based on the Oxford Levels of Evidence assessment as well as level of agreement after anonymised voting by the expert committee.

\section{Recommendation 1:}

Patients presenting with arthritis (any joint swelling, associated with pain or stiffness) should be referred to, and seen by, a rheumatologist, within 6 weeks after the onset of symptoms.

This recommendation is almost identical to its 2007 counterpart, but with subtle changes in the wording. After 2005, two studies have confirmed that patients with inflammatory arthritis in general, and those with suspected RA in particular, should be referred to rheumatologists as early as possible. ${ }^{19}{ }^{20}$ A delay in referral is one of the most important causes of late diagnosis and late start of effective treatment. Patients with early arthritis referred to a specialist within 3 months show better outcomes in terms of drug-free remission, radiographic damage and (less) need for orthopaedic surgery than those with late referral. ${ }^{15}$ This is also fully in line with standards of care developed for patients with RA and quality indicators as established by European Expert committees. ${ }^{21}$ On the basis of these data as well as the clinical experience of the committee members, it was recommended that diagnosis and start of treatment, both by a rheumatologist, should be established within a relatively short
Table 1 Updated EULAR recommendations for management of early arthritis, with LoE, GoR and LoA

\begin{tabular}{|c|c|c|c|c|}
\hline & & LoE* & GoR $^{*}$ & LoA* \\
\hline A. & Shared decision & na & na & $9.87 \pm 0.46$ \\
\hline B. & Rheumatologists & na & na & $9.78 \pm 0.67$ \\
\hline C. & Diagnosis & na & na & $9.78 \pm 0.67$ \\
\hline 1. & Early referral & $\mathrm{lb}$ & B & $9.43 \pm 1.16$ \\
\hline 2. & Clinical examination & Ilb & C & $9.48 \pm 0.99$ \\
\hline 3. & Prognosis & Ilb & C & $9.83 \pm 0.49$ \\
\hline 4. & Early treatment start & la & A & $9.35 \pm 1.07$ \\
\hline 5. & MTX, the anchor drug & la & A & $9.52 \pm 0.99$ \\
\hline 6. & NSAIDs & IV & D & $9.00 \pm 1.13$ \\
\hline 7. & Glucocorticoids & la & A & $9.00 \pm 1.28$ \\
\hline 8. & Remission and treatment strategies & $\mathrm{lb}, \mathrm{IV} \dagger$ & $A, D$ & $9.52 \pm 0.9$ \\
\hline 9. & Regular monitoring & la, IV & $A, D \ddagger$ & $9.13 \pm 1.06$ \\
\hline 10. & Non-pharmaceutical interventions & la & B & $8.96 \pm 1.26$ \\
\hline 11. & Prevention & Illb, IV & $C, D \ddagger$ & $8.96 \pm 1.19$ \\
\hline 12. & Patient information & $\mathrm{la}, \mathrm{lb}$ & B & $9.35 \pm 0.98$ \\
\hline
\end{tabular}

* $\mathrm{LOE}$ and GoR are based on the recommendations of the Oxford Centre for

Evidence-Based Medicine. LoA was based on an anonymised email voting system with a $0-10$ scale by all members of the expert committee (data are mean \pm SD; $100 \%$ of voters).

tThe general statement is evidence-based.

$¥$ The place in the treatment algorithm is based on expert consensus.

EULAR, European League Against Rheumatism; GoR, grade of recommendation; LoA level of agreement; LoE, level of evidence; MTX, methotrexate; na, not applicable; NSAIDs, non-steroidal anti-inflammatory drugs.

period after the onset of complaints which justifies the wording 'within 6 weeks' in this recommendation.

Joint swelling not due to trauma or bony swelling suggests early inflammatory arthritis, especially if associated with pain and morning stiffness $>30$ min. ${ }^{22}$ Several referral questionnaires evaluating swelling, pain and stiffness have been developed to aid in the detection of early arthritis. ${ }^{15}$ These questionnaires have a good sensitivity (86\%-90\%) and specificity $(90 \%)$, but have been tested only in small patient samples and lack confirmation in independent validation cohorts. The committee was of the opinion that an appropriately validated tool to help general practitioners in adequately diagnosing and referring patients 
with early arthritis is currently lacking. The strength of this recommendation was considered 'good' (category B) (table 1).

Recommendation 2:

Clinical examination is the method of choice for detecting arthritis, which may be confirmed by ultrasonography (US).

The expert committee unanimously appreciated the pivotal role of clinical examination. Clinical examination is still the cornerstone of detecting synovitis. This appreciation does not preclude that imaging modalities may be more sensitive in the detection of synovitis. US, including power Doppler techniques, may suggest synovitis by showing thickening of the synovial membrane, bursae and/or tendon sheaths with enhanced vascularity. ${ }^{15}$ Several controlled studies have suggested a greater sensitivity of US than clinical examination in detecting synovitis in the knee and in small joints. US has been evaluated in detail in the 'EULAR recommendations for the use of imaging of the joints in the clinical management of rheumatoid arthritis'. ${ }^{23}$ The expert committee did not recommend a more prominent role for US in the detection of synovitis, since it was broadly felt that potentially decreased specificity and lack of knowledge regarding the long-term consequences of positive US in individual patients did not currently justify a more prominent position for US. Furthermore, wording specifically referring to power Doppler was deleted, because the group considered that power Doppler should be part of every US joint examination anyway.

MRI has also been suggested to be more sensitive than clinical examination in the early detection of synovitis, ${ }^{23-25}$ but may face a lack of specificity as suggested by the prevalence of MRI abnormalities in the normal population. ${ }^{26}$ In contrast with US, which is now a common tool in many rheumatologist practices, the long scanning time, limited access and the relatively high costs limit the widespread use of MRI. Therefore, the expert committee considered that MRI should be proposed only in very difficult cases or in patients with specific forms of arthritis, and that further research is needed to better determine the place of this imaging modality in the diagnosis of patients with early arthritis. MRI was part of the 2007 recommendations but was deleted from the current set.

Recommendation 3:

If a definite diagnosis cannot be reached and the patient has early undifferentiated arthritis, risk factors for persistent and/or erosive disease, including number of swollen joints, acute-phase reactants, RF, ACPA and imaging findings, should be considered in management decisions.

This recommendation was slightly rephrased because the group wanted to highlight that early undifferentiated arthritis should be clearly differentiated from early RA. In addition, 'imaging' was used instead of 'radiographic' to show that imaging modalities other than plain radiographs may provide prognostic information. For patients with early arthritis, after the exclusion of specific forms of arthritis, the working diagnosis is often undifferentiated arthritis. The next step in the diagnostic procedure is to evaluate the risk of persistent and/or erosive arthritis, usually corresponding to the definition of RA, in an individual patient. ${ }^{27}$ This prognostic typing is now considered crucial to guide the optimal therapeutic strategy.

Since the 2007 exercise, many observational studies have evaluated the prognostic value of laboratory and imaging procedures for early arthritis. Most prognostic factors were analysed in a multivariate manner in these studies, to test their independent contribution. Commonly tested dependent variables were persistence, erosiveness or radiographic progression.

In most of the studies, ACPA and RF positivity and ACPA and RF levels have shown some predictive value for the development of persistent and erosive arthritis. This observation was clearly highlighted by EULAR and ACR since ACPAs, in addition to RF, have obtained an important weight in the 2010 ACR/EULAR classification criteria for RA. ${ }^{27} 28$ In addition, several recent studies have confirmed the independent association of ACPAs with a diagnosis of RA as well as with radiographic progression in patients with early arthritis. ${ }^{29-33} \mathrm{RF}$ has been assigned a similar weight as ACPAs in the 2010 ACR/ EULAR classification criteria for RA, although recent publications stemming from early arthritis cohorts and observational studies have suggested a lower predictive and diagnostic value of RF compared with ACPAs but RF has a stronger association with disease activity independent of the presence of ACPA. ${ }^{15}$ The combination of RF and ACPAs does not provide additional value to RF or ACPAs alone. ${ }^{28}$ In addition to ACPA, the number of swollen joints and the level of CRP and ESR are independent contributory factors.

Early erosion typical of RA is still a major prognostic factor in early arthritis and automatically leads to a classification of RA. $^{27}{ }^{34}$ Synovitis and erosion detected by MRI or US may predict further joint damage in early arthritis, but false positivity has been reported. ${ }^{26} 35$ MRI-detected bone marrow oedema and osteitis are independent predictors of radiographic progression in early RA, ${ }^{23}{ }^{24}$ but data are limited in early arthritis. Finally, two recent studies have shown that hand flexor or extensor tenosynovitis on $\mathrm{US}^{36}$ or $\mathrm{MRI}^{25}$ may be a specific-although not very sensitive-marker for RA classification.

Several combinations of diagnostic markers have been evaluated, but no one has been formally validated. ${ }^{15}$ In addition, multibiomarker tests have been proposed to evaluate disease activity, prognosis and response to therapy, but current data are not convincing and further research is warranted. ${ }^{15}$ Finally, it has been reported that substituting MRI for clinical examination in the $2010 \mathrm{ACR} / \mathrm{EULAR}$ criteria increases the sensitivity but decreases the specificity for a diagnosis of RA. ${ }^{15} \mathrm{MRI}$ is therefore of limited value in making a diagnosis of RA and is not recommended as a standard procedure.

Recommendation 4:

Patients at risk of persistent arthritis should be started on DMARDs as early as possible (ideally within 3 months), even if they do not fulfil classification criteria for an inflammatory rheumatologic disease.

This recommendation was slightly reworded and reiterates the unanimous opinion of the committee that an early treatment start is pivotal in the management of patients with early chronic arthritis such as early RA, early PsA or those at risk to develop persistent and erosive disease. The wording 'RA' is not used in this statement, but the implicit meaning is that persistent and/or erosive disease is factually synonymous to RA (see previous item) and justifies an early start with DMARDs. A new element is the maximum delay of 3 months after the onset of symptoms before starting the first DMARD. The expert committee was of the opinion that this time frame constitutes a 'window of opportunity' that should be considered to provide an optimal outcome in the patients at risk. Eight recent studies have endorsed an early treatment start. Four studies showed that introducing DMARDs within 3 months after the onset of symptoms leads to better outcome (remission, response to treatment, Health Assessment Questionnaire disability score or radiographic progression). ${ }^{37-40}$ Very recently, van Nies et $a l^{41}$ have suggested, based on data in the Etude et Suivi des Polyarthrites Indifferenciées Recentes (ESPOIR) and Leiden early arthritis cohorts, that 12-14 weeks represent an appropriate window within which therapy should be started in order to prevent 
arthritis persistence. In addition, disease duration at the time of DMARD initiation was the most important determinant of response to DMARD therapy in another study. ${ }^{15}$ This statement may raise questions about the best definition for 'early RA'. A duration of 3 months after the onset of symptoms may be the longest allowable delay in prescribing the first DMARD. However, this maximum delay is still difficult to meet in daily practice, while most of the recent 'early RA cohorts' allowed a delay of 6 months from the onset of symptoms (joint swelling usually) for inclusion. ${ }^{28} 2941$ A delay of not more than 6 months was also proposed in recent RA guidelines. ${ }^{42}$ A delay of more than 1 year from symptom onset must not be considered 'early' anymore.

Recommendation 5:

Among the DMARDs, methotrexate (MTX) is considered the anchor drug and unless contraindicated, should be part of the first treatment strategy in patients at risk of persistent disease.

This recommendation (previously no. 9) remains almost unchanged. Previous SLRs have confirmed the clinical and structural efficacy as well as the good safety profile of MTX. ${ }^{4} 4344$ An important argument to consider MTX an anchor drug as part of the first treatment strategy in patients at risk of persistent arthritis (eg, at risk of RA) is its good efficacy in early RA, and its 'practicability', both as monotherapy and in combination with glucocorticoids (GC), other csDMARDs and bDMARDs. ${ }^{4} 1345$ Recent trials in early DMARD-naïve patients with RA have evaluated MTX monotherapy versus csDMARDs combined with different dosages and routes of administration of GC. Verschueren et $a l^{46}$ have recently reported similar 16-week remission rates in high-risk patients with early RA receiving MTX monotherapy, MTX plus sulfasalazine (SSZ) or MTX plus leflunomide (LEF), all in combination with high-dose prednisone bridging strategies. In another trial, MTX plus temporary high-dose prednisone was not less effective than MTX plus SSZ plus temporary high-dose prednisone after 26 weeks. ${ }^{47}$ The Treatment in the Rotterdam Early Arthritis CoHort (tREACH) trial suggested short-lived superiority of MTX combined with SSZ, hydroxychloroquine and GC versus MTX and GC, but this superiority was not seen in all aspects, was not clinically meaningful and did ultimately not sustain after 1 year. ${ }^{48}$ The Treatment of Early Aggressive Rheumatoid Arthritis (TEAR) trial did not support a benefit of an intensive csDMARDs combination regimen over MTX monotherapy either. ${ }^{49}$ In the absence of clear signals for superiority of a csDMARDs combination regimen, and guided by a trend towards lower tolerability for csDMARD combination, ${ }^{16}$ the committee was of the opinion that the first treatment strategy should be MTX monotherapy with or without short-term high-dose GC as bridging therapy for most patients. In that regard, dose optimisation is an important aspect of first-line DMARD strategy, as previously reported $^{4} 45$ (MTX should be titrated rapidly to $20-30 \mathrm{mg} /$ week, depending on clinical response and tolerability; parenteral administration should be considered in case of inadequate clinical response or intolerance).

The superiority of bDMARDs plus MTX over MTX monotherapy has been proven in many randomised controlled trials (RCTs) and was confirmed by eight recent studies in the current SLR. ${ }^{16}$ In addition, two targeted synthetic DMARDs have recently demonstrated superiority to MTX, both used as monotherapy, in patients with early RA. ${ }^{50}$ Nevertheless, because the benefit-to-risk ratio of these biological and targeted synthetic DMARDs was not convincingly favourable in patients with early disease, because tight monitoring is anyway part of the current treatment strategy to identify those in need of adding biologics and also because of their high cost, the expert committee considered their use as a first treatment strategy inappropriate, except in rare situations.

Recent RCTs comparing other csDMARDs with MTX were lacking. The clinical efficacy of LEF, and to a lesser extent SSZ, is similar to MTX in established and recent RA. ${ }^{9}$ LEF is as effective as MTX in slowing radiographic damage, and its therapeutic maintenance is similar to that of MTX. ${ }^{9}$ In contrast, SSZ may be inferior to LEF and MTX in the long term. Although formal evidence prioritising MTX over other csDMARDs as the first DMARD used in early arthritis and/or early RA is lacking, the expert committee does recommend MTX as first-choice treatment (unless contraindicated) in patients at risk of persistent disease. LEF and (to a lesser extent) SSZ are considered the best alternatives. Of note, SSZ is considered safe during pregnancy in contrast to MTX and LEF. Finally, the committee is of the opinion that antimalarial drugs, which have shown less clinical efficacy and may not retard radiographic progression in patients with RA but may have positive metabolic effects, can be considered as partner in combination therapy or as DMARD monotherapy in patients with mild disease and comorbidities or with persistent arthritis other than RA. ${ }^{52}$

Recommendation 6:

Non-steroidal anti-inflammatory drugs (NSAIDs) are effective symptomatic therapies, but should be used at the minimum effective dose for the shortest time possible, after evaluation of gastrointestinal, renal and cardiovascular risks.

The SLR did not yield new data on NSAIDs in patients with early arthritis. The expert committee felt that symptomatic therapy with NSAIDs is still of value in patients presenting with early arthritis, but only after a careful consideration of gastrointestinal, renal and cardiovascular contraindications. In addition to the previous item no. 7 about NSAIDs, the group now reinforces the need to follow the US Food and Drug Administration and European Medicines Agency guidelines about NSAIDs, which includes wording about the shortest possible treatment duration, the minimum effective dose and the contraindications for patients at risk (http://www.fda.gov; http:// www.ema.europa.eu).

Recommendation 7:

Systemic GC reduce pain, swelling and structural progression, but in view of their cumulative side effects, they should be used at the lowest dose necessary as temporary ( $<6$ months) adjunctive treatment. Intra-articular glucocorticoid injections should be considered for the relief of local symptoms of inflammation.

The expert committee has intensively debated the role of GC in the management of early arthritis. This discussion was based on expert opinion and on new information obtained by the SLR. ${ }^{16}$ Recently, one meta-analysis of 14 RCTs in patients with RA and 2 RCTs in patients with 'early RA' has confirmed that systemic GC improve clinical and radiographic outcomes. ${ }^{16} 5354$ Preferably, therapy with systemic GC is temporary because of the risk of side effects, including weight gain, hypertension, diabetes, cataracts and osteoporosis, which justify careful monitoring and appropriate prevention. New data stemming from registries, observational studies and extensions of RCTs have also suggested an increased risk of severe infections, cardiovascular events and mortality. ${ }^{16}{ }^{55-60}$ In addition, there is evidence that intra-articular steroids may be an effective adjunct to DMARDs in relieving joint symptoms in patients presenting with early arthritis and may improve disease activity up to 24 months. ${ }^{16}$

The committee has reworded this item (no. 8 in the previous recommendations) in order to highlight the effectiveness of 
systemic GC for relieving symptoms and disease progression but also in order to point to the risks of cumulative side effects in the medium to long term. The committee is of the opinion that GC can only be justified if used at the lowest possible cumulative dose, for the shortest possible duration and exclusively as adjunct (or bridge) therapy to csDMARDs. GC monotherapy may mask disease activity before a diagnosis has been established and should be avoided in patients with early arthritis, in order to expedite a proper diagnosis, and secure an adequate prognosis and a prompt DMARD treatment start. Despite a fierce debate, this recommendation was finally approved by $95 \%$ of the members and obtained a high level of agreement (mean of 9.00 \pm 1.28 ) with anonymous voting. The wording 'low dose' and the optimal regimen (low daily dose or high dose then stepdown or parenteral boosts) in early arthritis are still under debate and will be mentioned in the research agenda (box 2).

Recommendation 8:

The main goal of DMARD treatment is to achieve clinical remission, and regular monitoring of disease activity, adverse events and comorbidities should guide decisions on choice and changes in treatment strategies to reach this target.

The 2007 recommendations for patients with early arthritis were among the first guidelines to highlight clinical remission as the main objective in the care of these patients. In the past 10 years, accumulating data have supported this as a major goal for the treatment of RA and other inflammatory arthritides. $^{6} 9111361$

The expert committee has decided to keep the wording of the previous recommendation no. 10 unchanged. A few new studies have confirmed that achieving clinical remission as early as possible results in better clinical outcomes and quality of life, and helps to prevent further structural damage, functional disability and job loss in patients with early arthritis and early RA. ${ }^{62}$ Which particular remission criteria should be used in practice remains unclear. Composite scores (disease activity score (DAS), DAS28, Clinical Disease Activity Index, Simplified Disease Activity Index (SDAI)) should be used, and the ACR-EULAR remission criteria (Boolean or SDAI) is likely the most stringent. ${ }^{63}$ An interesting definition for daily practice is 'the absence of signs and symptoms of significant inflammatory disease activity'. ${ }^{11}$ Recent evidence has suggested that remission leads to a better outcome than low disease activity (LDA), ${ }^{626465}$ and the committee was of the opinion that clinical remission according to the ACR-EULAR Boolean or indexbased definition is the target for every patient presenting with early arthritis. A LDA state could be an appropriate alternative goal only in cases in which remission is considered unfeasible. In this respect, factors such as comorbidities, age or adverse events must be considered, and may determine the desired treatment target, which will form the basis for the process of shared decision making with the patient.

The expert committee also discussed whether imaging remission should be included in the target, as suggested by some recent recommendations. ${ }^{23}$ Studies have suggested that ongoing inflammation seen by US, and to a lesser extent by MRI, in patients with clinical remission may predict structural progression. However, the significance thereof and its clinical utility are questionable and is associated with significant overtreatment and thus potential waste of societal resources; ${ }^{66}$ the SLR did not yield new information. ${ }^{15} 16$ Therefore, the expert committee suggested that the value of imaging remission should be part of the research agenda.

Finally, the committee felt that disease activity should be closely monitored in order to allow a timely change in DMARD
Box 2 Research agenda for management of early

arthritis

Diagnosis and prognosis

1. Which tools could help general practitioners to diagnose early arthritis and prioritise referral?

2. Can we better define the diagnostic and prognostic value of ultrasonography in early arthritis?

3. Can we better define the diagnostic and prognostic value of MRI in early arthritis?

4. What is the diagnostic value of the systematic screening of antinuclear antibodies in early arthritis?

5. Which new biomarkers/multibiomarkers may help to better evaluate disease activity, the prognosis and treatment response in early arthritis?

Treatment and outcome

1. Can we develop prediction models to better define the therapeutic strategy in early arthritis?

2. Can we define at what level of risk (for developing persistent arthritis) different pharmacological interventions have a favourable benefit-to-risk ratios?

3. Do combinations of csDMARDs provide a better benefit-torisk ratio than csDMARD monotherapy in early arthritis?

4. Can we better define 'low dose' and 'short term' use of glucocorticoids for an optimal medium-term to long-term benefit-to-risk ratio?

5. What is the optimal regimen (low daily dosage or high dose then step-down, or parenteral boosts) of glucocorticoids for better outcome in early arthritis?

6. Does imaging remission have an added benefit to clinical remission in treatment decisions?

7. What is the optimal interval at which to monitor radiographic progression in early chronic inflammatory arthritis?

8. What is the effectiveness of different non-pharmacological interventions in early arthritis?

9. Can physical activity/exercise reduce cardiovascular risk in early chronic arthritis?

10. Which study designs can best be used to investigate the comparative effectiveness and cost-effectiveness of different therapeutic strategies?

11. Is smoking cessation, oral hygiene, diets or psychological interventions beneficial for the outcome of patients with early arthritis?

12. What are the most efficient and effective information and education interventions and exercise programmes for early arthritis?

csDMARDs, conventional synthetic disease-modifying anti-rheumatic drugs; DMARD, disease-modifying antirheumatic drug.

therapy when necessary. The benefits of the treat-to-target approach have now amply been shown in patients with RA and PsA $^{11} 67$ and there is no reason to assume that the situation is different for early arthritis.

Recommendation 9:

Monitoring of disease activity should include tender and swollen joint counts, patient's and physician's global assessments, ESR and CRP, usually by applying a composite measure. Arthritis activity should be assessed at 1-month to 3 -month intervals until the treatment target has been reached. 
Radiographic and patient-reported outcome measures, such as functional assessments can be used to complement disease activity monitoring.

In every patient with active arthritis, closely monitoring disease activity is now considered of particular importance in the therapeutic strategy to provide a good outcome and this is highlighted by all of the most recent recommendations. ${ }^{6} 911134261$ Monitoring disease activity should be as frequent as the level of disease activity mandates, usually every 1-3 months, then potentially less frequently (such as every 6-12 months) once the treatment target has been achieved.

Nevertheless, three changes were proposed to this item (previously no. 12). First, a composite measure was recommended as the method of choice to monitor disease activity; second, a specific time frame for monitoring structural damage was deliberately left out and third, patient-reported outcomes were expanded beyond functional assessments.

Swollen joint count and progression of joint damage have been consistently found to be associated. ${ }^{68} 69$ In addition, many trials have supported the use of a tight control of disease activity assessed via composite measures that include joint count evaluation. $^{11} 166770$ Although it is difficult to formally investigate, the expert committee was of the opinion that monitoring the occurrence of radiographic progression is useful in view of one of the key objectives of managing early arthritis: the prevention of joint destruction. The determination of an optimal window for monitoring progression was added as an item for the research agenda (box 2).

Finally, patient-reported outcomes such as quality of life, fatigue and physical function are key to evaluate outcome ${ }^{71} 72$ and the committee has mandated them as part of disease monitoring.

\section{Recommendation 10:}

Non-pharmacological interventions, such as dynamic exercises and occupational therapy, should be considered as adjuncts to drug treatment in patients with early arthritis.

This recommendation has remained almost unchanged. The efficacy of non-pharmacological therapy has not been investigated in early arthritis and can only be extrapolated from the results of several RCTs in established RA. Hydrotherapy in patients with RA has been evaluated in some studies, ${ }^{73} 74$ but with insufficient evidence to support a strong recommendation; consequently, hydrotherapy was not included in the current statement but may be considered at the individual patient level. Previous RCTs have shown that joint-specific dynamic exercises may improve strength and physical function in RA, but the current SLR identified some controversial effects on disease activity. $^{16} 74$ Occupational therapy may improve functional ability and self-management but does not have a positive effect on disease activity; recent studies were not found. ${ }^{75}$

Finally, psychological counselling can be considered in selected patients, but trials investigating the efficacy of psychological interventions are lacking, and the committee did not include counselling in the statement. Furthermore, the SLR did not identify appropriate trials that evaluated the effectiveness of diets.

Since dynamic exercises, occupational therapy and to a lesser extent hydrotherapy have been associated with symptom relief in patients with established RA, the expert committee has decided to include them as adjunct therapies to pharmaceutical therapies in patients with early arthritis.

Recommendation 11:

In patients with early arthritis, smoking cessation, dental care, weight control, assessment of vaccination status and management of comorbidities should be part of overall patient care.
This recommendation is new and largely based on expert opinion. The expert committee felt that during the last decade evidence has accumulated that highlights the importance of the management of comorbidities (eg, cardiovascular diseases, metabolic conditions (eg, hyperlipidaemia, diabetes), lung diseases, infections, malignancies, osteoporosis and depression) in the context of the management of early arthritis. ${ }^{76-82}$ Comorbidities may affect life expectancy and outcomes (physical function, quality of life) independently of disease activity in patients with inflammatory arthritis. In addition, coexisting diseases may affect the efficacy and safety of antirheumatic therapies. ${ }^{82}$ Obesity and smoking may affect the response to treatment in inflammatory arthritis. ${ }^{80}$ Prevention is now considered key in the management of chronic inflammatory rheumatic diseases, but comorbidities are still not optimally managed. ${ }^{76}$ Smoking is the best-established modifiable risk factor in the development of RA and spondyloarthritis. ${ }^{83}{ }^{84}$ Furthermore, tobacco use has been associated with the presence of extra-articular manifestations such as rheumatoid nodules and also serum RF and ACPAs. While smoking does not seem to be associated with the perpetuation of disease activity or progression of RA, ${ }^{85}$ it may affect the outcome of spondyloarthritis. ${ }^{84}$

$\mathrm{RA}$ is associated with periodontal disease, although the direction of the relationship still remains unclear. ${ }^{86}$ The microbiome may play a role in chronic arthritis risk and progression, and Porphyromonas gingivalis infection could promote aberrant citrullination and a local breach of tolerance to citrullinated peptides. The potentially beneficial contribution of oral hygiene has been put on the research agenda.

Although current data do not prove that risk-factor modification is beneficial to patients, the modifiable risk factors identified in the SLR are so generic in nature that the committee was unanimously of the opinion that a recommendation aiming at abolishing their potential influence on arthritis (and general health) would not harm patients and may convey some benefits.

In addition, the expert committee noted that fewer patients with chronic arthritis than recommended are currently vaccinated, ${ }^{87}$ and that this should be specifically mentioned.

\section{Recommendation 12:}

Patient information concerning the disease, its outcome (including comorbidities) and its treatment is important. Education programmes aimed at coping with pain, disability, maintenance of ability to work and social participation may be used as adjunct interventions.

This recommendation was very similar to the previous item no. 6. Obviously, full transparency about the disease and its treatment options should be an integral part of the management of any chronic disease, and constitutes the core of overarching principle A. Other healthcare providers share the responsibility in the provision of information. Studies have suggested that adherence to treatment is dependent on the quality of information exchange and the quality of the interaction between the patient and healthcare professionals, including rheumatologists. ${ }^{16}$

EULAR has recently recommended that 'people with inflammatory arthritis should have access to and be offered patient education throughout the course of their disease, including as a minimum, at diagnosis, at pharmacological treatment change and when required by the patient's physical or psychological condition'. 88 The content and delivery of patient education should be individually tailored, with individual and group sessions representing different approaches to delivery. It is impossible to prioritise a single educational intervention since all tested interventions have only short-term benefits and feature 
cross-national and cultural variations. ${ }^{16}$ Improved quality of life is a major aim for patients and the committee proposed to add 'social participation' as one of the objectives of these education programmes. The expert committee also felt that patients should be aware that comorbidities may affect the outcome and treatment of inflammatory arthritis, and that their screening and management should be part of the global management of early arthritis.

\section{DISCUSSION}

The update of the EULAR recommendations for the management of early arthritis followed the 2014 EULAR Standardised Operating Procedures. ${ }^{14}$ The committee has proposed an important revision of the items, but obviously most major recommendations have remained intact. These updated recommendations for management of early arthritis contain 3 overarching principles, 12 recommendations and 2 algorithms that integrate all the recent developments in the management of early arthritis. The definition of the term 'management' was unchanged and includes all spectra of management of early arthritis, including referral, diagnosis, prognosis, classification, information, education, nonpharmacological interventions and pharmacological treatments and monitoring of the disease. The term 'early arthritis' was restricted to 'early inflammatory arthritis' and mainly, but not only, focused on the risk of chronic arthritis.

The expert committee had to face a limitation in that most of the published data on treatment and strategy on which they could build their recommendations involved studies in patients with early RA or established RA, rather than specific studies of early arthritis. Despite this limitation, the committee considered much of the data for early RA sufficiently robust and relevant for extrapolating to 'early arthritis with a certain propensity to become persistent.' The scope was different compared with the EULAR recommendations for the management of RA, ${ }^{13}$ which focussed on the use of DMARDs in both early and established disease. However, there are overlaps with regard to the first-line therapy for early arthritis at risk of persistence (figure 1) and for early RA (DMARD-naïve and usually $<6$ months disease duration). Not surprisingly, the two sets of recommendations are very congruent on these specific points.

These recommendations have important strengths including the composition of the expert committee comprising 20 rheumatologists, including 2 research fellows, from 12 European countries and new addition of 1 healthcare professional and 2 patient representatives. The committee chose to grade the level of evidence provided by every study, which was based on the methodology of the study, and took this grading into consideration when discussing the content and the strength of the recommendations. An important consideration in the discussions was always whether the type of study fitted the content of the research question that was at the basis of the literature search. The recommendations were based on the most recent evidence and on expert opinion. For example, the expert committee felt that evidence supported comorbidities as possibly affecting the outcome of arthritis and also treatment efficacy and safety and should be considered in the management of all early arthritis cases. Despite the sparse evidence, the expert committee also wanted to indicate that smoking cessation and dental care could be proposed to patients with early arthritis, and that both patients and healthcare professionals should be aware of the importance to improve vaccination coverage. In this respect, a new recommendation on prevention was added (item no. 11). Of note, the level of agreement among the experts was high for each item (means of 9.0-9.9), which support the appropriateness and validity of the recommendations.

In light of the current literature and despite important recent advances, the committee felt that further development of new tools is needed for early and accurate diagnosis and prognosis, including new biomarkers, better understanding of the added value of US and MRI and creation of prediction algorithms for long-term outcome (box 2). Finally, the expert committee felt that the comparative effectiveness and cost-effectiveness of the different strategic modalities in early arthritis, including the effectiveness of non-pharmacological interventions, need additional research.

While these 'recommendations' are deliberately not called 'guidelines', they do reflect a strong view of many European experts including patient representatives. They should provide rheumatologists, general practitioners, medical students, healthcare professionals, health authorities and patients a practical approach to the management of early arthritis, even though each healthcare professional should choose the most appropriate management strategy for each individual patient. To that end, it is hoped that the recommendations will be widely disseminated and discussed within the community of rheumatologists and other healthcare professionals caring for patients with early arthritis and that they will help improve the standard of care for patients with arthritis across different healthcare systems. Obviously, these recommendations will probably need amendment after about 5 years to incorporate new scientific evidence.

\section{Author affiliations}

${ }^{1}$ Rheumatology Department, Lapeyronie Hospital, Montpellier University, UMR 5535, Montpellier, France

${ }^{2}$ Department of Clinical Immunology \& Rheumatology, Amsterdam Rheumatology Center, Amsterdam and Zuyderland Medical Centre, Heerlen, The Netherlands

${ }^{3}$ Division of Rheumatology, Department of Medicine 3, Medical University of Vienna, Vienna, Austria

${ }^{4}$ Biological Therapies Unit, Servicio de Reumatología. Hospital Universitario de la Princesa, IIS-IP, Madrid, Spain

${ }^{5}$ EULAR Standing Committee of People with Arthritis/Rheumatism in Europe (PARE), Zurich, Switzerland

${ }^{6}$ Division of Physiotherapy, Department of Neurobiology Care Sciences and Society, Karolinska Institutet, Huddinge, Sweden

${ }^{7}$ Department of Orthopaedics, Danderyd Hospital, Stockholm, Sweden

${ }^{8}$ Department of Rheumatology and Clinical Immunology, Charité-University Medicine Berlin, Berlin, Germany

${ }^{9}$ Department of Rheumatology, Center for Rheumatic Diseases, University of Medicine and Pharmacy, Bucharest, Romania

${ }^{10}$ Department of Rheumatology, Centre for Arthritis and Rheumatic Diseases, St Vincent's University Hospital, Dublin Academic Medical Centre, Dublin, Ireland

${ }^{11}$ Medicine Faculty, APHP, Rheumatology B Department, Paris Descartes University, Cochin Hospital, Paris, France

${ }^{12}$ Leeds NIHR Musculoskeletal Biomedical Research Unit, LTHT, Leeds Institute of Rheumatic and Musculoskeletal Medicine, University of Leeds, Leeds, UK

${ }^{13}$ Fondazione Policlinico Gemelli Academic Hospital, Catholic University School of Medicine, Rome, Italy

${ }^{14}$ Rheumatology Department, Hospital de Santa Maria, Lisbon Academic Medical

Centre, Lisbon

${ }^{15}$ Instituto de Medicina Molecular, Faculdade de Medicina Universidade de Lisboa,

Portugal

${ }^{16}$ Rheumatology Research Group, Institute of Inflammation and Ageing, University of Birmingham, Birmingham, UK

${ }^{17}$ Sandwell and West Birmingham Hospitals NHS Trust, Birmingham, UK

${ }^{18}$ Rheumatology Department, Complexo Hospitalario Universitario de Ferrol, A

Coruña, Spain

${ }^{19}$ Faculty of Medicine, Department of Rheumatology, University of Debrecen,

Debrecen, Hungary

${ }^{20}$ Department of Rheumatology, Diakonhjemmet Hospital, Oslo, Norway

${ }^{21}$ Department of Rheumatology, Leiden University Medical Center, Leiden, The Netherlands

${ }^{22}$ Department of Rheumatology, Erasmus Medical Center, Rotterdam, The Netherlands

${ }^{23}$ Department of Clinical Immunology \& Rheumatology, Academic Medical Center, Amsterdam, The Netherlands 
Correction notice This article has been corrected since it published Online First. The legend for figure 1 has been updated.

Acknowledgements We thank Louise Falzon, Columbia University Medical Centre (USA), expert librarian for her contribution in the systematic literature research.

Contributors $B C$ and RL wrote the first draft. CID and $\mathrm{CH}$ performed the systematic literature review. All authors have participated in the generation of the recommendations, have revised critically the manuscript and approved the final version.

Funding This initiative was supported by an unrestricted grant from the European League Against Rheumatism.

Competing interests All the participants in this initiative have disclosed conflict of interest. The individual declaration of conflict of interest is available on demand at the European League Against Rheumatism secretariat and is summarised below: BC has received honoraria from BMS, Janssen, Eli Lilly, Merck, Novartis, Pfizer, Roche-Chugai, Sanofi and UCB, and research grants from Pfizer, Roche-Chugai and UCB. RL has received honoraria and/or research grants from AbbVie, Ablynx, Amgen, AstraZeneca, Bristol-Myers Squibb, Celgene, Janssen, Galapagos, GlaxoSmithKline (GSK), Novartis, Novo-Nordisk, Merck, Pfizer, Roche, Schering-Plough, TiGenix, UCB. RL is director of Rheumatology Consultancy BV. CID has received honoraria from BMS, Merck, Pfizer, Roche-Chugai and UCB. DA has received honoraria from AbbVie, BMS, Centocor, Janssen, Eli Lilly, Medac, Merck, Pfizer, Roche and UCB. JMA-G has received honoraria from AbbVie, BMS, MSD, Novartis, Pfizer, Roche, Sanofi and UCB, and research grants from MSD, Pfizer, Roche and UCB. GRB has received honoraria from AbbVie, BMS, Janssen, Eli Lilly, MSD, Novartis, Pfizer, Roche-Chugai and UCB, and research grants from Pfizer, Roche-Chugai and UCB. CC has received speaker and consulting fees from AbbVie, Amgen, Angellini, AstraZeneca, Bristol-Myers Squibb, Egis, MSD, Pfizer, Richter, Roche, Sanofi, Servier, Teva, UCB, Zentiva. RC has received honoraria from AbbVie, Roche, Pfizer and Amgen. MD has received honorarium fees for participating at advisory boards or symposia organised by Pfizer, AbbVie, UCB, Roche, Eli Lilly, Novartis, Sanofi, Merck, BMS. His department has received research grants from Pfizer, AbbVie, UCB, Roche, Eli Lilly, Novartis, Sanofi, Merck, BMS. PE has provided expert advice for AbbVie, AstraZeneca, BMS, Eli Lilly, Merck, Novartis, Pfizer, Roche, Sandoz, Samsung, UCB. GF has received honoraria from AbbVie, Pfizer, MSD, Roche, Janssen, Eli Lilly, UCB and research grants from Roche, MSD. JF has received unrestricted research grants or acted as a speaker for AbbVie, Amgen, BMS, Biogen, Celltrion, Celgene, Hospira, Janssen, MSD, Novartis, Novo-Nordisk, Pfizer, Roche, UCB. KR has received honoraria from AbbVie, Pfizer, Roche and Janssen and research grants from AbbVie. JSS has received grants for his institution from AbbVie, Janssen, Eli Lilly, MSD, Pfizer, Roche and has provided expert advice to and/or had speaking engagements for AbbVie, Amgen, AstraZeneca, Astro, Celgene, Celltrion, GSK, ILTOO, Janssen, Eli Lilly, Medimmune, MSD, Novartis-Sandoz, Pfizer, Roche, Samsung, Sanofi, UCB. ZS has received consultant and speakers fees from AbbVie, MSD, Bristol-Myers Squibb, Roche, Pfizer. TKK has received fees for speaking and/or consulting from Biogen, BMS, Boehringer Ingelheim, Celltrion, Eli Lilly, Epirus, Hospira, Merck-Serono, Novartis, Orion Pharma, Pfizer, Sandoz and UCB. RvV has received research support and grants from AbbVie, Amgen, BMS, GSK, Pfizer, Roche, UCB, and honoraria for consultancy from AbbVie, Biotest, BMS, Celgene, Crescendo, GSK, Janssen, Eli Lilly, Merck, Novartis, Pfizer, Roche, UCB, Vertex.

Provenance and peer review Not commissioned; externally peer reviewed.

\section{REFERENCES}

1 Machado P, Castrejon I, Katchamart W, et al. Multinational evidence-based recommendations on how to investigate and follow-up undifferentiated peripheral inflammatory arthritis: integrating systematic literature research and expert opinion of a broad international panel of rheumatologists in the 3E Initiative. Ann Rheum Dis 2011;70:15-24

2 Huizinga TWJ, Machold KP, Breedveld FC, et al. Criteria for early rheumatoid arthritis: from Bayes' law revisited to new thoughts on pathogenesis. Arthritis Rheum 2002:46:1155-9.

3 Combe B. Early rheumatoid arthritis: strategies for prevention and management. Best Pract Res Clin Rheumatol 2007;21:27-42.

4 Gaujoux-Viala C, Nam J, Ramiro S, et al. Efficacy of conventional synthetic disease-modifying antirheumatic drugs, glucocorticoids and tofacitinib: a systematic literature review informing the 2013 update of the EULAR recommendations for management of rheumatoid arthritis. Ann Rheum Dis 2014;73:510-15.

5 Smolen JS, Aletaha D, Koeller M, et al. New therapies for treatment of rheumatoid arthritis. Lancet 2007;370:1861-74.

6 Gossec L, Smolen JS, Ramiro S, et al. European League Against Rheumatism (EULAR) recommendations for the management of psoriatic arthritis with pharmacological therapies: 2015 update. Ann Rheum Dis 2016;75:499-510.

7 Nam JL, Ramiro S, Gaujoux-Viala C, et al. Efficacy of biological disease-modifying antirheumatic drugs: a systematic literature review informing the 2013 update of the EULAR recommendations for the management of rheumatoid arthritis. Ann Rheum Dis 2014;73:516-28.
8 Avci $A B$, Feist $E$, Burmester G-R. Biologicals in rheumatoid arthritis: current and future. RMD Open 2015;1:e000127.

9 Combe B, Landewe $R$, Lukas $C$, et al. EULAR recommendations for the management of early arthritis: report of a task force of the European Standing Committee for International Clinical Studies Including Therapeutics (ESCISIT). Ann Rheum Dis 2007:66:34-45.

10 Monti S, Montecucco C, Bugatti S, et al. Rheumatoid arthritis treatment: the earlier the better to prevent joint damage. RMD Open 2015;1(Suppl 1):e000057.

11 Smolen JS, Breedveld FC, Burmester GR, et al. Treating rheumatoid arthritis to target: 2014 update of the recommendations of an international task force. Ann Rheum Dis 2016;75:3-15.

12 Smolen JS, Landewé R, Breedveld FC, et al. EULAR recommendations for the management of rheumatoid arthritis with synthetic and biological disease-modifying antirheumatic drugs. Ann Rheum Dis 2010;69:964-75.

13 Smolen JS, Landewé $\mathrm{R}$, Breedveld FC, et al. EULAR recommendations for the management of rheumatoid arthritis with synthetic and biological disease-modifying antirheumatic drugs: 2013 update. Ann Rheum Dis 2014;73:492-509.

14 van der Heijde D, Aletaha D, Carmona L, et al. 2014 Update of the EULAR standardised operating procedures for EULAR-endorsed recommendations. Ann Rheum Dis 2015;74:8-13.

15 Hua $C$, Daien $\mathrm{Cl}$, Combe B. Diagnosis, prognosis and classification of early arthritis: results of a systematic literature informing the 2016 update of the EULAR recommendations for the management of early arthritis. RMD Open.

16 Daïen $\mathrm{Cl}$, Hua $\mathrm{C}$, Combe $\mathrm{B}$, et al. Pharmacologic and non-pharmacologic therapies in early arthritis: results of a systematic literature informing the 2016 update of the EULAR recommendations for the management of early arthritis. RMD Open 2016.

17 OCEBM Levels of Evidence. CEBM. 2016 (cited 3 May 2016). http://www.cebm.net/ ocebm-levels-of-evidence/

18 Bykerk V, Emery P. Delay in receiving rheumatology care leads to long-term harm. Arthritis Rheum 2010;62:3519-21.

19 van der Linden MPM, le Cessie S, Raza K, et al. Long-term impact of delay in assessment of patients with early arthritis. Arthritis Rheum 2010:62:3537-46.

20 Feldman DE, Bernatsky S, Houde $\mathrm{M}$, et al. Early consultation with a rheumatologist for RA: does it reduce subsequent use of orthopaedic surgery? Rheumatology 2013;52:452-9.

21 Stoffer MA, Smolen JS, Woolf A, et al. Development of patient-centred standards of care for rheumatoid arthritis in Europe: the eumusc.net project. Ann Rheum Dis 2014;73:902-5

22 Emery $\mathrm{P}$, Breedveld FC, Dougados $\mathrm{M}$, et al. Early referral recommendation for newly diagnosed rheumatoid arthritis: evidence based development of a clinical guide. Ann Rheum Dis 2002;61:290-7.

23 Colebatch AN, Edwards CJ, Østergaard M, et al. EULAR recommendations for the use of imaging of the joints in the clinical management of rheumatoid arthritis. Ann Rheum Dis 2013:72:804-14.

24 Duer-Jensen A, Hørslev-Petersen $\mathrm{K}$, Hetland ML, et al. Bone edema on magnetic resonance imaging is an independent predictor of rheumatoid arthritis development in patients with early undifferentiated arthritis. Arthritis Rheum 2011;63: 2192-202.

25 Nieuwenhuis WP, Krabben A, Stomp W, et al. Evaluation of magnetic resonance imaging-detected tenosynovitis in the hand and wrist in early arthritis. Arthritis Rheumatol 2015:67:869-76.

26 Mangnus L, Schoones JW, van der Helm-van Mil AHM. What is the prevalence of MRI-detected inflammation and erosions in small joints in the general population? A collation and analysis of published data. RMD Open 2015;1:e000005.

27 Aletaha D, Neogi T, Silman AJ, et al. 2010 Rheumatoid arthritis classification criteria: an American College of Rheumatology/European League Against Rheumatism collaborative initiative. Ann Rheum Dis 2010;69:1580-8.

28 Funovits J, Aletaha D, Bykerk V, et al. The 2010 American College of Rheumatology/European League Against Rheumatism classification criteria for rheumatoid arthritis: methodological report phase I. Ann Rheum Dis 2010;69:1589-95.

29 Mouterde G, Lukas C, Logeart I, et al. Predictors of radiographic progression in the ESPOIR cohort: the season of first symptoms may influence the short-term outcome in early arthritis. Ann Rheum Dis 2011;70:1251-6.

30 van den Broek M, Dirven L, Klarenbeek NB, et al. The association of treatment response and joint damage with ACPA-status in recent-onset RA: a subanalysis of the 8-year follow-up of the BeSt study. Ann Rheum Dis 2012;71:245-8.

31 Hetland ML, Stengaard-Pedersen $\mathrm{K}$, Junker $\mathrm{P}$, et al. Radiographic progression and remission rates in early rheumatoid arthritis-MRI bone oedema and anti-CCP predicted radiographic progression in the 5-year extension of the double-blind randomised CIMESTRA trial. Ann Rheum Dis 2010;69:1789-95.

32 Degboé $Y$, Constantin A, Nigon D, et al. Predictive value of autoantibodies from anti-CCP2, anti-MCV and anti-human citrullinated fibrinogen tests, in early rheumatoid arthritis patients with rapid radiographic progression at 1 year: results from the ESPOIR cohort. RMD Open 2015;1:e000180.

33 Akdemir G, Verheul MK, Heimans L, et al. Predictive factors of radiological progression after 2 years of remission-steered treatment in early arthritis patients: a post hoc analysis of the IMPROVED study. RMD Open 2016;2:e000172. 
34 van der Heijde D, van der Helm-van Mil AHM, Aletaha D, et al. EULAR definition of erosive disease in light of the 2010 ACR/EULAR rheumatoid arthritis classification criteria. Ann Rheum Dis 2013;72:479-81.

35 Rezaei $H$, Torp-Pedersen $S$, af Klint $E$, et al. Diagnostic utility of musculoskeletal ultrasound in patients with suspected arthritis-a probabilistic approach. Arthritis Res Ther 2014;16:448.

36 Sahbudin I, Pickup L, Cader Z, et al. OP0015 ultrasound-defined tenosynovitis is a strong predictor of early rheumatoid arthritis. Ann Rheum Dis 2015;74(Suppl 2):69-70.

37 Bosello S, Fedele AL, Peluso G, et al. Very early rheumatoid arthritis is the major predictor of major outcomes: clinical ACR remission and radiographic non-progression. Ann Rheum Dis 2011;70:1292-5.

38 Gremese E, Salaffi F, Bosello SL, et al. Very early rheumatoid arthritis as a predictor of remission: a multicentre real life prospective study. Ann Rheum Dis 2013;72:858-62.

39 Lukas C, Combe B, Ravaud P, et al. Favorable effect of very early disease-modifying antirheumatic drug treatment on radiographic progression in early inflammatory arthritis: data from the Étude et Suivi des polyarthrites indifférenciées récentes (study and follow-up of early undifferentiated polyarthritis). Arthritis Rheum 2011;63:1804-11.

40 Söderlin MK, Bergman S, BARFOT Study Group. Absent "Window of Opportunity" in smokers with short disease duration. Data from BARFOT, a multicenter study of early rheumatoid arthritis. J Rheumatol 2011;38:2160-8.

41 van Nies JAB, Tsonaka R, Gaujoux-Viala C, et al. Evaluating relationships between symptom duration and persistence of rheumatoid arthritis: does a window of opportunity exist? Results on the Leiden early arthritis clinic and ESPOIR cohorts. Ann Rheum Dis 2015;74:806-12.

42 Singh JA, Saag KG, Bridges SL, et al. 2015 American College of Rheumatology guideline for the treatment of rheumatoid arthritis. Arthritis Rheumatol 2016;68:1-26.

43 Ramiro S, Gaujoux-Viala C, Nam JL, et al. Safety of synthetic and biological DMARDs: a systematic literature review informing the 2013 update of the EULAR recommendations for management of rheumatoid arthritis. Ann Rheum Dis 2014:73:529-35

44 Ramiro S, Smolen JS, Landewé R, et al. Pharmacological treatment of psoriatic arthritis: a systematic literature review for the 2015 update of the EULAR recommendations for the management of psoriatic arthritis. Ann Rheum Dis 2016:75:490-8.

45 Visser K, Katchamart W, Loza E, et al. Multinational evidence-based recommendations for the use of methotrexate in rheumatic disorders with a focus on rheumatoid arthritis: integrating systematic literature research and expert opinion of a broad international panel of rheumatologists in the $3 \mathrm{E}$ Initiative. Ann Rheum Dis 2009:68:1086-93.

46 Verschueren P, De Cock D, Corluy L, et al. Methotrexate in combination with other DMARDs is not superior to methotrexate alone for remission induction with moderate-to-high-dose glucocorticoid bridging in early rheumatoid arthritis after 16 weeks of treatment: the CareRA trial. Ann Rheum Dis 2015:74:27-34.

47 den Uyl D, ter Wee M, Boers $M$, et al. A non-inferiority trial of an attenuated combination strategy ('COBRA-light') compared to the original COBRA strategy: clinical results after 26 weeks. Ann Rheum Dis 2014;73:1071-8.

48 de Jong PH, Hazes JM, Han HK, et al. Randomised comparison of initial triple DMARD therapy with methotrexate monotherapy in combination with low-dose glucocorticoid bridging therapy; 1-year data of the tREACH trial. Ann Rheum Dis 2014;73:1331-9.

49 Moreland LW, O'Dell JR, Paulus HE, et al. A randomized comparative effectiveness study of oral triple therapy versus etanercept plus methotrexate in early aggressive rheumatoid arthritis: the treatment of Early Aggressive Rheumatoid Arthritis Trial. Arthritis Rheum 2012;64:2824-35.

50 Lee EB, Fleischmann $\mathrm{R}$, Hall $\mathrm{S}$, et al. Tofacitinib versus methotrexate in rheumatoid arthritis. N Engl J Med 2014:370:2377-86.

51 Fleischmann R, Schiff M, van der Heijde D. Baricitinib, Methotrexate, or Combination in Patients with Rheumatoid Arthritis and No or Limited Prior DiseaseModifying Antirheumatic Drug Treatment. Arthritis Rheumatol 2016 Oct 9. doi:10. 1002/art.39953.

52 Katz SJ, Russell AS. Re-evaluation of antimalarials in treating rheumatic diseases: re-appreciation and insights into new mechanisms of action. Curr Opin Rheumatol 2011:23:278-81

53 Verschueren P, De Cock D, Corluy L, et al. Patients lacking classical poor prognostic markers might also benefit from a step-down glucocorticoid bridging scheme in early rheumatoid arthritis: week 16 results from the randomized multicenter CareRA trial. Arthritis Res Ther 2015;17:97.

54 Seegobin SD, Ma MHY, Dahanayake C, et al. ACPA-positive and ACPA-negative rheumatoid arthritis differ in their requirements for combination DMARDs and corticosteroids: secondary analysis of a randomized controlled trial. Arthritis Res Ther 2014;16:R13.

55 Dixon WG, Abrahamowicz M, Beauchamp M-E, et al. Immediate and delayed impact of oral glucocorticoid therapy on risk of serious infection in older patients with rheumatoid arthritis: a nested case-control analysis. Ann Rheum Dis 2012;71:1128-33.
56 del Rincón I, Battafarano DF, Restrepo JF, et al. Glucocorticoid dose thresholds associated with all-cause and cardiovascular mortality in rheumatoid arthritis. Arthritis Rheumatol 2014;66:264-72.

57 Listing J, Kekow J, Manger B, et al. Mortality in rheumatoid arthritis: the impact of disease activity, treatment with glucocorticoids, TNF $\alpha$ inhibitors and rituximab. Ann Rheum Dis 2015;74:415-21.

58 Roubille $C$, Richer $V$, Starnino $T$, et al. The effects of tumour necrosis factor inhibitors, methotrexate, non-steroidal anti-inflammatory drugs and corticosteroids on cardiovascular events in rheumatoid arthritis, psoriasis and psoriatic arthritis: a systematic review and meta-analysis. Ann Rheum Dis 2015;74: 480-9.

59 Ajeganova S, Svensson B, Hafström I. Low-dose prednisolone treatment of early rheumatoid arthritis and late cardiovascular outcome and survival: 10-year follow-up of a 2-year randomised trial. BMJ Open 2014;4:e004259.

60 de Hair M. Long-Term Adverse Events after Daily Concomitant Treatment with 10mg Prednisone in the 2-Year Computer Assisted Management in Early Rheumatoid Arthritis Trial-II. ACR Meeting Abstracts [cited 10 May 2016].

61 Smolen JS, Braun J, Dougados M, et al. Treating spondyloarthritis, including ankylosing spondylitis and psoriatic arthritis, to target: recommendations of an international task force. Ann Rheum Dis 2014;73:6-16.

62 Combe B, Logeart I, Belkacemi MC, et al. Comparison of the long-term outcome for patients with rheumatoid arthritis with persistent moderate disease activity or disease remission during the first year after diagnosis: data from the ESPOIR cohort Ann Rheum Dis 2015;74:724-9.

63 Felson DT, Smolen JS, Wells G, et al. American College of Rheumatology/European League Against Rheumatism provisional definition of remission in rheumatoid arthritis for clinical trials. Ann Rheum Dis 2011;70:404-13.

64 Ruyssen-Witrand A, Guernec G, Nigon D, et al. Aiming for SDAl remission versus low disease activity at 1 year after inclusion in ESPOIR cohort is associated with better 3-year structural outcomes. Ann Rheum Dis 2015;74:1676-83.

65 Radner H, Smolen JS, Aletaha D. Remission in rheumatoid arthritis: benefit over low disease activity in patient-reported outcomes and costs. Arthritis Res Ther 2014;16: R56.

66 Dale J, Stirling A, Zhang R, et al. Targeting ultrasound remission in early rheumatoid arthritis: the results of the TaSER study, a randomised clinical trial. Ann Rheum Dis 2016;75:1043-50.

67 Coates LC, Moverley AR, McParland L, et al. Effect of tight control of inflammation in early psoriatic arthritis (TICOPA): a UK multicentre, open-label, randomised controlled trial. Lancet 2015:386:2489-98.

68 Klarenbeek NB, Güler-Yüksel M, van der Heijde DMFM, et al. Clinical synovitis in a particular joint is associated with progression of erosions and joint space narrowing in that same joint, but not in patients initially treated with infliximab. Ann Rheum Dis 2010;69:2107-13.

69 Gärtner M, Sigmund IK, Alasti F, et al. Clinical joint inactivity predicts structural stability in patients with established rheumatoid arthritis. RMD Open 2016;2: e000241.

70 Akdemir G, Markusse IM, Dirven L, et al. Effectiveness of four dynamic treatment strategies in patients with anticitrullinated protein antibody-negative rheumatoid arthritis: a randomised trial. RMD Open 2016;2:e000143.

71 van Steenbergen HW, Tsonaka R, Huizinga TWJ, et al. Fatigue in rheumatoid arthritis; a persistent problem: a large longitudinal study. RMD Open 2015;1: e000041.

72 Gossec L, Dougados M, Dixon W. Patient-reported outcomes as end points in clinical trials in rheumatoid arthritis. RMD Open 2015;1:e000019.

73 Verhagen AP, Bierma-Zeinstra SM, Boers M, et al. Balneotherapy (or spa therapy) for rheumatoid arthritis. An abridged version of Cochrane Systematic Review. Eur J Phys Rehabil Med 2015;51:833-47.

74 Hurkmans E, van der Giesen FJ, Vliet Vlieland TP, et al. Dynamic exercise programs (aerobic capacity and/or muscle strength training) in patients with rheumatoid arthritis. Cochrane Database Syst Rev 2009;(4):CD006853.

75 Ekelman BA, Hooker L, Davis A, et al. Occupational therapy interventions for adults with rheumatoid arthritis: an appraisal of the evidence. Occup Ther Health Care 2014:28:347-61

76 Dougados M, Soubrier M, Antunez A, et al. Prevalence of comorbidities in rheumatoid arthritis and evaluation of their monitoring: results of an international, cross-sectional study (COMORA). Ann Rheum Dis 2014;73:62-8.

77 Baillet A, Gossec L, Carmona L, et al. Points to consider for reporting, screening for and preventing selected comorbidities in chronic inflammatory rheumatic diseases in daily practice: a EULAR initiative. Ann Rheum Dis 2016:75:965-73.

78 Peters MJL, Symmons DPM, McCarey D, et al. EULAR evidence-based recommendations for cardiovascular risk management in patients with rheumatoid arthritis and other forms of inflammatory arthritis. Ann Rheum Dis 2010;69:325-31.

79 Gherghe AM, Dougados M, Combe B, et al. Cardiovascular and selected comorbidities in early arthritis and early spondyloarthritis, a comparative study: results from the ESPOIR and DESIR cohorts. RMD Open 2015;1: e000128 
80 Daïen Cl, Sellam J. Obesity and inflammatory arthritis: impact on occurrence, disease characteristics and therapeutic response. RMD Open 2015;1:000012.

81 Bacconnier L, Rincheval N, Flipo R-M, et al. Psychological distress over time in early rheumatoid arthritis: results from a longitudinal study in an early arthritis cohort. Rheumatology 2015;54:520-7.

82 Radner H, Yoshida K, Mjaavatten MD, et al. Development of a multimorbidity index: impact on quality of life using a rheumatoid arthritis cohort. Semin Arthritis Rheum 2015:45:167-73.

83 Sparks JA, Karlson EW. The roles of cigarette smoking and the lung in the transitions between phases of preclinical rheumatoid arthritis. Curr Rheumatol Rep 2016;18:15.

84 Poddubnyy D, Haibel H, Listing J, et al. Cigarette smoking has a dose-dependent impact on progression of structural damage in the spine in patients with axial spondyloarthritis: results from the German SPondyloarthritis Inception Cohort (GESPIC). Ann Rheum Dis 2013;72:1430-2.

85 Vesperini V, Lukas C, Fautrel B, et al. Association of tobacco exposure and reduction of radiographic progression in early rheumatoid arthritis: results from a French multicenter cohort. Arthritis Care Res 2013;65:1899-906.

86 Fuggle NR, Smith TO, Kaul A, et al. Hand to mouth: a systematic review and meta-analysis of the association between rheumatoid arthritis and periodontitis. Front Immunol 2016:7:80.

87 Hua C, Morel J, Ardouin E, et al. Reasons for non-vaccination in French rheumatoid arthritis and spondyloarthritis patients. Rheumatology (Oxford) 2015;54:748-50.

88 Zangi HA, Ndosi M, Adams J, et al. EULAR recommendations for patient education for people with inflammatory arthritis. Ann Rheum Dis 2015;74:954-62. 